\title{
Autour de La Fronde : femmes, techniques et inventions au XIXème siècle
}

\author{
La Fronde: women, technology and invention in the 19th Century
}

\author{
Anne Chanteux ${ }^{1}$ \\ ${ }^{1}$ EHESS, laboratoire Koyré, anne.chanteux@lecnam.net
}

\begin{abstract}
RESUME. À la fin du XIXème siècle parait le journal La Fronde qui portera une attention particulière aux sciences et techniques de son époque. Le journal de Marguerite Durand publiera même des listes de brevets déposés par des femmes. Qui sont ces femmes ? Comment ont-elles pu intervenir sur le terrain de la technique alors que leur éducation et leurs droits les prédisposaient à de toutes autres activités?

ABSTRACT. At the end of the 19th Century, the journal La Fronde focused particular attention on the science and technology of its time. This journal, founded by Marguerite Durand, published lists of patents filed by women. Who were these women? How did they intervene in the field of technology when their education and rights predisposed them to other activities?

MOTS-CLES. brevet, XIXème siècle, histoire des techniques, histoire des femmes, invention.

KEYWORDS. patent, 19th Century, history of technology, history of women, invention.
\end{abstract}

\section{Introduction}

"L'humanité ne doit aux femmes aucune découverte signalée, pas même une invention utile » (Marie d'Agout, Opinions de femmes sur la femme, Revue encyclopédique, 28 novembre 1896).

Le génie est masculin, c'est entendu. Nous pourrions multiplier à l'envi les citations, de femmes comme d'hommes, déclinant les faiblesses de la femme, ses incapacités et limites. Le long XIXe siècle a produit de nombreux discours autour de «la » femme, sa physiologie, son caractère, sa place dans la société, la famille. Dans le même temps les femmes sortent de leurs cadres habituels : si la bourgeoise se rencogne dans son habitat, un grand nombre de femmes rejoint dans les usines et les ateliers les cohortes de travailleurs. « Ouvrière, mot impie », clame alors Michelet. Mais comme le souligne le titre du livre de Sylvie Schweitzer : les femmes ont toujours travaillé [SCH 02]. Si la forme du travail évolue (davantage de travail à l'extérieur du foyer), le fait subsiste : les femmes travaillent depuis longtemps, participent à l'économie domestique, à l'économie nationale en produisant des biens, des services.

Le récit historique écrit par les hommes a « oublié » ces femmes et cette histoire, nourrie par les hauts faits des grands hommes a pu étayer par la suite les discours sur l'invisibilité des femmes [PER 12] et la nature cachée de leur production [KHA 15]. L'histoire des femmes s'est heurtée à des problèmes de sources : les statistiques sont rarement sexuées et la grande histoire a longtemps été privilégiée au détriment de la "petite », de l'intime, du quotidien. Or la femme n'apparait pas dans la sphère publique, elle est en retrait. Son histoire s'inscrit en quelque sorte " en creux », selon l'expression de Françoise Thébaud [THE 07]. On la retrouve dans les archives, les statistiques, les textes en déchiffrant les vides et en comparant les sources entre elles.

Parmi ces sources, ressort la présence singulière du journal La Fronde. Singulière par son dessein, son lectorat, sa volonté affichée de faire en tout comme les journaux masculins et de ne pas se cantonner à un public féminin. Dans le champ de l'histoire des techniques, le principal intérêt du journal créé par Marguerite Durand, outre le fait qu'il est entièrement rédigé par des femmes, est l'attention qu'il porte aux nouveautés scientifiques et techniques à travers ses articles, ses brèves, 
ses rubriques mais aussi et surtout la publication de listes de brevets déposés par des femmes qu'il entreprend à partir de 1900.

Selon Zorina Khan, historienne de l'économie, «[...] les archives des brevets, quoiqu'imparfaites, fournissent la source la plus objective et la moins spéculative de connaissances au sujet des femmes et de l'invention » [KHA 05] et, bien que les brevets ne représentent pas toute l'activité inventive, ils permettent de mettre en lumière une activité méconnue : la relation entre femme et technique, femme et invention.

Mis à part le travail de Zorina Khan dans le champ de l'histoire économique, il n'existe pas d'étude des brevets pris par des femmes en France, pas plus que d'interrogations sur leur implication dans le champ de l'invention. Aborder le sujet par une analyse statistique des dépôts de brevets peut éclairer un pan de l'histoire des techniques laissé en friche.

Avant d'examiner de plus près les brevets listés dans La Fronde, il convient de situer le degré d'acculturation technique des femmes dans une époque peu propice à leur accorder une éducation scientifique et technique. Se pose alors la question de comprendre comment des femmes ont-elles pu se présenter comme inventrices dans une société qui leur accorde si peu de droits et de crédit?

\section{Situation de la femme du XIXe au regard de la technique}

«On a vu des femmes très savantes comme il en fut de guerrières, mais il n'y en a jamais eu d'inventrices » (Voltaire, Questions sur l'Encyclopédie, 1771)

\subsection{Une éducation technique limitée}

Avant 1850, l'éducation dispensée aux filles ne se distingue pas de celle du XVIIIe siècle. La majorité des filles est éduquée à la maison ou dans les couvents. Elles peuvent aussi passer par des écoles, payantes ou non, mais la scolarité ne dure que 2 ou 3 années ${ }^{1}$. L'enseignement reçu : la couture, l'entretien du ménage. Pour les jeunes bourgeoises, des arts d'agréments en plus (peinture, musique).

A partir de la $2^{\text {ème }}$ moitié du XIXe, des avancées importantes ont lieu, du premier baccalauréat attribué en 1861 à une jeune femme, Julie-Victoire Daubié, à l'ouverture aux filles des principales écoles techniques en 1917 et, en 1924, à l'unification de l'enseignement secondaire masculin et féminin. Des combats auront lieu tout au long de ce siècle pour réclamer une éducation digne de ce nom mais il faut bien reconnaître que l'éducation des filles au XIXe siècle n'est qu'un très pâle reflet de l'éducation dispensée aux garçons (notamment du point de vue des sciences et des techniques) et qu'elle consiste essentiellement à en faire de bonnes épouses et de bonnes mères : «L'égalité d'éducation, c'est l'unité reconstituée dans la famille » [FER 93]. Et même si elles peuvent assister à des cours et conférences publics, les femmes n'accèdent pas à un véritable enseignement scientifique et technique.

Faute d'un enseignement adéquat, comment les femmes peuvent-elles comprendre et maîtriser des processus techniques puis agir sur eux ? Joël Lebeaume, spécialiste de la didactique de la technologie, propose une explication à l'imprégnation technique qu'ont vraisemblablement reçue certaines femmes : le travail manuel, organisé autour de « cinq travaux considérés comme la base des travaux féminins : la couture usuelle, le raccommodage, le tricot, le crochet et la marque » [LEB

\footnotetext{
${ }^{1}$ Martine SONNET, I'Education des filles à l'époque moderne, HAL, 12 décembre 2011
} 
99] assure une relation avec « les sciences appliquées et les arts appliqués » [LEB 99] Selon lui, une des conséquences positives de l'enseignement des pratiques domestiques, spécifiquement dispensé aux filles, est qu'il ne peut se faire sans un apprentissage minimum des sciences naturelles, physiques et chimiques (étude de l'eau, de l'air, de l'hygiène) [LEB 99]. Le rapport du jury de l'Exposition universelle de 1900 relève aussi les notions scientifiques préparatoires à l'enseignement ménager: les trois états de la matière, l'air («action comburante, composition. Propriétés physiques »), le feu, etc [PIC 02].

Le programme des «Leçons de choses » présenté par des institutrices lors de l'Exposition de 1889 puis généralisé et adopté par les écoles maternelles de toutes les régions françaises, apporte des :

«[...] connaissances usuelles, à côté d'observations directes, comprennent de nombreuses «causeries » plus ou moins illustrées par l'image. Elles abordent la sécurité, l'hygiène [...]. Elles transportent l'esprit des enfants sur des choses qu'ils ne voient pas (baleines, mines d'or). Elles portent sur la connaissance des objets usuels grâce à des monographies descriptives mentionnant leur origine et leur usage, sur la connaissance des activités domestiques (confitures, aliments) ou des occupations (commerce, agriculture), ou sur la connaissance des prouesses techniques que sont à l'époque l'éclairage, les aéronefs, les voitures... » [LEB 99].

On peut voir là une des possibilités d'accès au savoir technique pour les deux sexes ou du moins d'éveil de la curiosité. Cependant cette éducation est un vernis technique qui ne peut à lui seul rendre les femmes autonomes dans une pratique rendue plus complexe par le développement accéléré des innovations.

L'enseignement professionnel offre des possibilités aux jeunes filles : par exemple les cours de comptabilité à l'usage des dames et demoiselles, fondés en 1857 et dirigés par Élise Luquin, qui accueillent une centaine d'élèves par an. Vanté par l'économiste Michel Chevalier, cette école élargit ses ambitions, étoffe sa liste de cours (plus de langues vivantes) et est rebaptisé École supérieure de commerce de jeunes filles vingt ans plus tard. En 1862, Elisa Lemonnier (Saint-Simonienne) ouvre sa première école afin de donner un savoir pratique technique et professionnel aux jeunes filles ; cet enseignement essaimera jusqu'à ouvrir plusieurs écoles dans toute la France. A Lyon, l'école de La Martinière des filles ouvre ses portes en 1879 autour des spécialités de dessin-broderie, de lingerie et de commerce. On remarquera l'aspect local des enseignements techniques liés à l'industrie régionale. Mais, avant 1917, aucune école technique de garçons, quel que soit le niveau requis pour l'admission, n'est accessible aux femmes.

\subsection{Comment acquérir des connaissances autrement?}

A partir du XIXe siècle, les revues, spécialisées ou de vulgarisation, permettent de s'informer sur les innovations et de se forger une bonne culture scientifique et technique, même si elles ne peuvent remplacer une véritable éducation dans ces domaines. Le support éditorial du XIXe représente un bon terreau pour les scientifiques et inventeurs non académiques, parmi lesquels figurent les femmes.

Livres et revues se multiplient et sont largement diffusés grâce aux progrès des techniques de l'imprimerie et des moyens de transports. Au début du XIXe une centaine de périodiques scientifiques et techniques est publiée dans le monde ; 10000 vers 1900 [JAC 15]. Les femmes peuvent accéder à ces supports d'information scientifique et technique (encore faut-il avoir de l'argent pour acheter les journaux ou pouvoir s'abonner à des cabinets de lecture). Il s'agit, pour un grand nombre de personnes, hommes et femmes, suivant le milieu social, d'un mode d'accès à un 
savoir impossible à acquérir autrement. Les «[...] dynamiques d'appropriation et d'acculturation technique [sont] rendues possibles par l'essor d'une littérature technique, commerciale ou académique, par les multiples formes de publicisation de la technique, qui «traduisent» les nouveautés pour un public large ou connaisseur, appelé à son tour à comprendre, interpréter, adapter les inventions » [HIL 06].

Par ailleurs, le XIXe siècle connaît un essor des journaux pour femmes : parfois militant, souvent familial et bien-pensant, le journal à lectorat féminin est un terrain riche pour la compréhension du rôle et de l'activité des femmes dans toutes les classes de la société et dans tous les domaines de l'activité. Mais peu s'intéressent aux techniques. Les journaux professionnels néanmoins permettent d'acquérir une culture technique : le Journal des sages-femmes (parution de 1873 à 1914) par exemple.

Des femmes participent à cet essor et écrivent, pas seulement dans les journaux qui leur sont dédiés. A la fin du siècle les magazines anglais comptent $12 \%$ de collaboratrices qui écrivent sur des sujets scientifiques (physique, microbiologie, aéronautique, technologie, ...) [BEN 97]. La lecture d'articles scientifiques et techniques écrits par des femmes a pu jouer un rôle d'émulation et inciter les femmes à se reconnaître des capacités en ces domaines. Qu'en est-il d'une influence de ce type en France?

Si les femmes n'ont pas d'accès à une éducation scientifique et technique, elles travaillent au sein de l'entreprise familiale ou à l'extérieur. Les veuves gèrent l'entreprise au décès du mari et, comme du temps des corporations, transmettent les savoir-faire. Elles sont porteuses de compétences qu'elles ont intégrées à leur pratique quotidienne et, en ce sens, elles sont bel et bien dépositaires d'un savoir technique.

\section{Les techniques dans La Fronde}

«L'humanité ne doit aux femmes aucune découverte industrielle, pas la moindre mécanique. [...] L'homme invente, perfectionne, travaille, produit et nourrit la femme. [...] elle n'a même pas inventé son fuseau et sa quenouille ». (Proudhon, Amour et mariage, 1876)

Créée en 1897 par Marguerite Durand, journaliste, La Fronde est le premier quotidien « rédigé, composé, administré uniquement par des femmes » sans être pour autant un journal féminin. Il traite tous les sujets, offre des rubriques politiques, économiques, sociales, scientifiques, littéraires.

Selon la directrice, «L'important est de montrer qu'en toutes matières, on pourrait confectionner un journal en réussissant aussi bien que les hommes » [RAB 96]. Si d'ailleurs, aucun homme n'est admis, ce n'est pas par sexisme : «Le fait d'écarter toute collaboration masculine n'était pas ostracisme contre les hommes, mais si un seul homme eût fait partie de la maison, même dans l'administration, on eût dit que le journal était fait dans les coulisses par des hommes et que les femmes signaient seulement » explique Marguerite Durand dans une lettre à une collaboratrice [DIZ 92].

Lancé le jeudi 9 décembre 1897, le journal est quotidien jusqu'au $1^{\text {er }}$ septembre 1903, paraissant 7 jours sur 7, y compris les jours fériés. D’octobre 1903 à mars 1905, il devient mensuel avant de s'arrêter. Il connaît une brève résurrection en 1914, sous forme de quotidien, puis de mai 1926 à mijuillet 1907. Il finit hebdomadaire de mi-juillet 1907 à juillet 1928. Un numéro exceptionnel et unique est consacré au centenaire de Clémence Royer, le 19 juin 1930.

Le premier numéro de La Fronde est tiré à plus de 200000 exemplaires, mais trois mois plus tard, le titre ne diffuse plus que 49000 exemplaires. En 1899, il tombe à 14600 . C'est pourquoi, 
soutenu à bouts de finances par sa directrice, il ne tiendra que jusqu'en 1903 sous forme de quotidien [RAB 96]. Pour estimer ces tirages par rapport aux grands titres de la presse politique parisienne, Le Journal des Débats tirait à 26000 exemplaires en 1910, Le Temps (auquel La Fronde a été comparé) à 35000 exemplaires en 1904, 45000 en 1912 [LEM 99].

Dès le premier mois de son existence, La Fronde octroie une place à la science et à la technique : du 9 (date du premier numéro) au 31 décembre 1897, deux brèves et deux articles dont un long (courant sur deux jours) sont consacrés à ces sujets. L'article daté du 16 décembre traite du progrès à travers l'histoire de «l'automobilisme aux XVIe et XVIIe siècles ». La chronique écrite par Dorothée Klumpke sur deux jours, les 25 et 26 décembre, est précise, historique et contemporaine à la fois : «l'astronomie et ses auxiliaires » décrit le travail dans un observatoire et les instruments utilisés. L'auteur en est une spécialiste, américaine d'origine : « en 1884, elle devint la première femme à obtenir un doctorat d'astronomie pour son étude des anneaux de Saturne. Elle dirigea ensuite le bureau de l'Observatoire de Paris chargé de collecter les observations photographiques destinées à la constitution du catalogue international d'étoiles établi par le Congrès international astronomique » [KLE 01].

Ce premier mois porte en germe ce que sera le journal durant son existence de quotidien et on retrouvera des sujets scientifiques et techniques, variés et traités de façon aussi diverse.

En 1898 et 1899, une rubrique régulière s'installe: A travers les sciences. En 1899, un supplément traite des nouvelles découvertes. A partir de 1900, des listes de brevets d'inventions déposés par des femmes sont publiées.

Parmi les quelques titres de la même période auxquels il peut être comparé par les idéaux et principes défendus (le Droit des femmes, la Femme de l'avenir, la Française, ...), aucun n'a traité les sciences et les techniques de leur époque comme l'a fait La Fronde : en créant des rubriques, en suivant de près l'actualité en ces domaines, qu'elle soit féminine ou pas, ce journal occupe une place originale. Un d'entre eux, la Revue scientifique des femmes: sciences physiques, naturelles, médicales, philosophiques et sociales, créée en 1888 par Céline Renooz, peut lui être comparé sur ce point. Mais il n'a duré que deux années (1888-1889) avec une parution trimestrielle. Une rubrique «les femmes inventrices » est présente deux fois, la première listant des brevets américains, la seconde évoquant deux inventions faites par des femmes, l'une française, l'autre américaine.

Il faut cependant relativiser la présence d'articles scientifiques et techniques : la volonté de la fondatrice était de faire un quotidien semblable aux autres pour appuyer, par le sérieux et la rigueur de son entreprise, les revendications qu'elle portait. Or le XIXe siècle consacre une large place aux discours sur les progrès scientifiques et techniques (journaux pour tous publics, pièces de théâtre, conférences, etc) et, puisque tous les journaux de cette époque proposaient un «feuilleton scientifique » ou une rubrique sur le même thème, rien d'étonnant à ce que La Fronde, journal généraliste rédigé pour un public mixte, fasse de même. Relevons aussi l'intérêt marqué par des revues du XVIIIe siècle pour l'industrialisation croissante : "l'un des thèmes principaux étant les inventions en technologie et sciences, le périodique décrit grand nombre de produits de l'art mécanique récemment inventés : le chauffage pour voiture, la bicyclette, les ponts, bateaux et meubles en fer, les douches, la machine à écrire, les voitures à moteur, le diorama, le chemin de fer, ... » [KLE 01]. Cet exemple est bien antérieur à la naissance du quotidien La Fronde mais prouve qu'il se situe dans une continuité et qu'il n'innove pas en s'intéressant aux domaines scientifiques et techniques.

En revanche le journal innove par la renommée et l'implication de ses journalistes. Nous avons déjà évoqué Dorothée Klumpke, il convient de citer également, outre Séverine et Clémence Royer qui ont prêté leur plume prestigieuse au journal, Blanche Galien, pseudonyme de Louise Napias 
ayant soutenu en 1900 d'une thèse en pharmacie sur l'action de la bactérie charbonneuse sur les hydrates de carbone, Aline Valette, inspectrice du travail et journaliste, auteur en 1898 d'une remarquable enquête sociale sur le travail des femmes ${ }^{2}$. Ces femmes seront des pionnières: premières journalistes à suivre la bourse, à entrer au Palais-Bourbon, dans les prétoires, à suivre les procès (notamment la révision de celui de Dreyfus à Rennes).

\section{Des brevets et des femmes}

«Au regard de la loi constitutionnelle, la première des femmes est un être inférieur au dernier des hommes » (Paul Valéry, Le suffrage des femmes, in : Revue de Paris, 15 février 1931)

«Le brevet d'invention est un titre délivré par le gouvernement pour conférer à un inventeur le droit exclusif et temporaire d'exploiter l'objet de sa découverte, sous certaines conditions. » [LAM 81]. Le dépôt d'un brevet suppose que l'inventeur souhaite protéger son invention et la produire ou la faire produire. D'ailleurs la loi l'oblige à mettre en exploitation son invention sous peine d'être déchu de tous ses droits. La démarche de dépôt d'un brevet suit des règles strictes (description de l'invention, dessin) et n'est pas gratuite : une taxe est demandée pour le dépôt et, lors de l'attribution du brevet, une somme dépendant de la durée de protection choisie est perçue par l'Etat. Des annuités doivent être versées par l'inventeur sous peine de déchéance de ses droits La France, contrairement à l'Allemagne et aux Etats-Unis, n'examine pas l'intérêt de la demande avant d'octroyer un brevet : les brevets sont «SGDG» (Sans Garantie Du Gouvernement) mais l'inventeur n'ayant pas créé ou apporté de perfectionnement significatif s'expose à la nullité de son dépôt et à la déchéance de ses droits s'il est avéré que la condition de nouveauté n'est pas respectée. Dans ces conditions, il faut être motivé, disposer immédiatement d'argent (plus ou moins selon qu'on dépose seul ou qu'on fait appel à un agent) et être prêt à investir commercialement. Conditions peu simples à réunir à une époque où le code Napoléon place la femme en position de tutelle juridique.

Face au développement des techniques, si les femmes sont en mesure d'acquérir des connaissances théoriques (par la lecture) et pratiques (par l'enseignement professionnel et leur propre pratique professionnelle), elles n'en sont pas moins limitées par les conditions juridiques de leur époque si jamais elles entreprennent d'y participer. Les réformes juridiques issues de la Révolution française ont entrouvert une fenêtre entre 1789 et 1804 mais l'instauration du code civil a eu tôt fait de la refermer. En termes légaux, la femme du XIXe est un être mineur placée sous la coupe de son mari ou de son père lorsqu'elle n'a pas accédé au rang d'épouse. Mais elle paie des impôts et peut aller en prison : en somme des devoirs mais pas de droits. Les statuts de célibataire (après 21 ans) et de veuve octroient cependant des droits spécifiques : droits civils identiques à ceux des hommes mais ils conservent tout de même certaines incapacités. Les veuves peuvent être chefs d'entreprises, dirigeantes d'exploitations et il n'est pas rare de croiser la mention « société veuve » parmi les titulaires de brevets ou dans les actes de sociétés.

L'article 1124 du code civil de 1804 stipule : « les personnes privées de droits juridiques sont les mineurs, les femmes mariées, les criminels et les débiles mentaux ». Ces personnes peuvent déposer un brevet mais elles ne peuvent l'exploiter. Là se situe une impossibilité majeure pour les femmes : «Elle doit obtenir l'autorisation de son mari pour chaque acte juridique et en toute affaire, que ce soit pour diriger son ménage ou pour exercer une activité commerciale indépendante. » [GER 16]. Seul un statut professionnel permet aux femmes de contracter des obligations sans autorisation maritale : celui des marchandes publiques (article 220).

\footnotetext{
2 Aline VALETTE, Marcelle CAPY, femmes et travail au XIXe siècle : enquêtes de La Fronde et la Bataille syndicaliste, Syros, 1984
} 
Le brevet d'invention «n'est [...] autre chose que la constatation du contrat qui est intervenu entre l'inventeur et la société : l'inventeur livre sa découverte à la connaissance de tous ; mais, en échange, il reçoit du gouvernement, représentant la société, la garantie d'un monopole temporaire, d'une jouissance exclusive placée sous la protection de la loi » [CON 84]. Une femme mariée déposant des brevets seule est donc reconnue comme contractante même si, au regard de la loi, « elle ne peut ester en justice ni contracter (article214-226) » [GER 16]. Armengaud spécifie que c'est aux tribunaux de décider « si l'impétrant peut légalement jouir du privilège du brevet, ou dans quelles limites il peut en jouir » [ARM 93].

Mais dès la fin du XVIIIe siècle « les actes invitent [...] à nuancer fortement l'idée d'une sujétion totale de l'épouse » [BEA 16]; au XIXe la pratique est plus souple que le code civil, souvent ignoré [SOH 96] et des femmes, veuves ou pas, ont pu disposer de leurs avoirs, parmi lesquels des brevets. Le brevet étant considéré comme un bien mobilier dont le propriétaire peut disposer de la même manière que ses autres biens, la femme peut en hériter en tant qu'ayant-droit : par exemple la veuve. Elle peut breveter des idées produites par ses employés ou ses parents. Et elle peut par la suite déposer d'autres brevets et accroître ainsi le capital de l'entreprise qu'elle perpétue.

Le fonctionnement du système des brevets dessine le portrait de l'inventrice : relativement autonome puisqu'elle peut disposer d'argent et être représentante légale, possédant un savoir technique puisqu'elle connaît la valeur de son invention et la juge digne d'un brevet, active socialement car elle intègre à son savoir celui des réseaux de protection et de valorisation de l'invention.

\section{Les brevets publiés dans La Fronde}

«[...] il est trois choses qui font la grandeur de l'esprit humain : raisonner, abstraire, généraliser, - trois choses auxquelles l'intelligence des femmes a, pour l'instant, quelque peine à se hausser. Et cela même nous explique pourquoi les hommes ont, plus que les femmes, le don de la découverte et le génie de l'invention. »(Charles Turgeon, le féminisme français. 1 : l'émancipation individuelle et sociale de la femme, 1902)

La Fronde commence à faire paraître des listes de brevets en 1900 (première liste le 18 mai) : « grâce à l'obligeance de MM. Marillier et Robelet (office international pour l'obtention des brevets d'inventions en France et à l'étranger, 42 boulevard Bonne Nouvelle, Paris), nous pourrons chaque mois faire connaître à nos lecteurs les brevets délivrés aux femmes » (La Fronde, vendredi 18 mai 1900). Cet office a adressé, le 27 avril 1900, une lettre à Marguerite Durand pour proposer l'envoi, tous les quinze jours ou tous les mois, de listes recensant « les derniers brevets français pris par des femmes » :

«Il arrive assez fréquemment en France que des brevets d'invention sont pris par des femmes dans toutes les industries, mais plus particulièrement dans celles qui les intéressent le plus directement, c'est-à-dire les vêtements, l'hygiène, l'alimentation, etc.

Nous venons vous demander si vous pensez qu'il serait intéressant pour vos lectrices d'être mises au courant de l'existence de ces brevets. En tout cas, cette communication ne pourrait, à notre avis, qu'être utile à l'œuvre d'émancipation que vous poursuivez. » (Lettre de MM. Marillier et Robelet à Mme Marguerite Durand, datée du 27 avril 1900. Bibliothèque Marguerite Durand, dossier documentaire sur les femmes et les inventions, DOS 600 INV). 
Jusqu'à la création de l'Office national de la propriété industrielle (ONPI) en 1901, qui réunira dans un même lieu les catalogues et les publications de brevets, il est difficile de consulter et de reproduire les brevets [EMP 02]. Les agences d'ingénieurs ont la charge de diffuser l'information technique : «Avant 1902, date à laquelle l'Etat prend enfin ses responsabilités en matière de publication de brevets d'invention, c'est en effet aux agents d'inventions qu'échoit la tâche de publier, partiellement, les brevets d'invention récents. » [GAL 06]. L'office de MM. Marillier et Robelet propose donc un service précieux en mettant à disposition des listes comprenant le numéro de brevet, la date de dépôt, le nom de la déposante, l'intitulé du brevet.

En échange de ce travail, les ingénieurs demandent «...simplement d'en indiquer la provenance au bas de chaque liste que vous publierez. ». En 1925 deux cabinets similaires (V. Prévost et T. Durand, et l'office Picard) proposeront le même service.

De ce premier courrier daté de 1900, on peut déduire que la publication des brevets ne vient pas des journalistes de La Fronde mais d'une proposition extérieure (et masculine !). Le journal tirant à l'époque environ à 14600 exemplaires (chiffres de 1899), on peut se demander quel bénéfice espérait l'office Marillier et Robelet en effectuant régulièrement le recensement des brevets déposés par des femmes. Quelles retombées pouvait-il attendre de la publication de son nom dans un journal à si petit tirage et à lectorat majoritairement féminin ? Est-ce la notoriété du journal, de ses collaboratrices qui a incité les directeurs à ce surcroît de travail ? Ont-ils fait une démarche semblable auprès des autres journaux féministes ? On peut le supposer puisque le journal La Femme de l'avenir publie également des listes en 1901. Le petit pourcentage que représentaient les brevets déposés par des femmes suffit-il à justifier cette implication ou ont-ils pensé, plus prosaïquement, que les femmes ayant des époux, ceux-ci pouvaient également être de futurs clients ? Nous n'avons pas la réponse de Marguerite Durand à ce premier courrier mais elle figure implicitement à travers la publication régulière des listes dans La Fronde.

«L'œuvre d'émancipation » que poursuit le journal passe par la publicité autour des réalisations faites par des femmes, les brevets en font partie. On peut voir une incitation à inventer dans la publication, le 9 septembre 1901, de l'arrêté du Ministère du commerce sur la rédaction des brevets. Le même jour, sur la même page, paraît l'annonce d'un concours de moteurs à alcool lancé par le Ministère de l'agriculture : aucun domaine n'est étranger aux compétences présumées des femmes.

En se référant aux statistiques de brevets déposés [EMP 02], on peut calculer le pourcentage de brevets féminins cités dans La Fronde, sachant que les listes publiées dans le quotidien ne sont pas exhaustives (La comparaison de ces listes avec le bulletin officiel de la propriété industrielle et commerciale (BOPI) à l'INPI fait apparaître quelques brevets supplémentaires, non recensés par le cabinet).

\begin{tabular}{|c|c|c|c|}
\hline & $\begin{array}{c}\text { Nb de brevets déposés } \\
\text { par des femmes et cités } \\
\text { dans la Fronde }\end{array}$ & $\begin{array}{c}\text { Nb total de brevets déposés } \\
\text { (source : INPI) }\end{array}$ & $\begin{array}{c}\text { \% de brevets féminins par } \\
\text { rapport au nombre total }\end{array}$ \\
\hline $\mathbf{1 9 0 0}$ & 119 & 12400 & $0,95 \%$ \\
\hline $\mathbf{1 9 0 1}$ & 136 & 12103 & $1,12 \%$ \\
\hline $\mathbf{1 9 0 2}$ & 102 & 12026 & $0,84 \%$ \\
\hline
\end{tabular}

(Les années 1899 et 1903 ont été omises volontairement, le nombre recensé dans le journal ne portant que sur un semestre chaque). 
L'année 1900 étant celle de l'Exposition Universelle, on peut estimer les chiffres de cette annéelà et de la suivante à l'aune de l'enthousiasme que provoqua cette manifestation.

Aux Etats-Unis, les femmes obtenaient 303 brevets en 1893, soit 1,5\% du total des brevets [TRE 79]. En 1898, il y eut en Angleterre 638 brevets pris par des femmes sur un total de 27639 dépôts [JAF 05]. Si on laisse de côté l'écart entre les deux pays lié à la tradition en matière de dépôt de brevets, plus ancienne en Angleterre qu'en France [HIL 00], le ratio anglais est tout de même nettement plus élevé : $2,30 \%$, soit plus du double de brevets déposés par des femmes. Il s'agit là d'une année exceptionnelle : «le pourcentage ne s'élève pas au-dessus de $2 \%$ après 1912 ou audessus de $1 \%$ après 1938. » [VAN 99]. Globalement, le pourcentage français semble plus faible. Le dynamisme des anglo-saxonnes en matière d'inventions peut s'expliquer par leur plus grande autonomie légale et leurs actions pionnières en matière de féminisme.

Le journal La Fronde présente un grand intérêt pour l'exploration des relations entre femmes et invention : publiant méthodiquement les brevets, il offre un corpus pour les étudier. L'information complémentaire apportée par les publications de brevets disponibles à l'Institut national de la propriété industrielle permet de situer ces brevets dans la production nationale et précise des données non fournies par le journal.

\section{Qui sont ces inventrices ?}

«Je crois qu'il ne manque aux femmes que les occasions de s'instruire et de prendre de l'émulation; on en voit assez qui se distinguent, malgré les obstacles de l'éducation et du préjugé, pour croire qu'elles ont autant d'esprit que la plupart des hommes qui acquièrent de la célébrité dans les sciences » (Jérôme Lalande, Astronomie des dames, 1785, reed. Paris, Bidauld, 1806).

531 brevets déposés entre le 3 juillet 1899 et le 12 mai 1903 ont été listés dans la Fronde du 18 mai 1900 au 19 août 1903.

Ils ont été pris par 461 inventrices : 50 d'entre elles ont déposé de 2 à 6 brevets chacune. Parmi ces «multi récidivistes», on trouve par exemple une artiste peintre, Consuelo Fould, épouse de Grasse, qui dépose 5 brevets tous relatifs à des corsets ; mais aussi Mme veuve Levassor qui, après la mort de son mari et avec M. de Boisse, dépose des brevets pour des carburateurs et autres régulateurs ; ou encore Mademoiselle Josepha Schiele résidant en Belgique, qui dépose 6 demandes dans la période étudiée (d'autres brevets seront ensuite demandés et obtenus) sur des bougies d'allumage pour moteurs à explosion mais aussi des procédés d'argenture et de dorure.

Sur un total de 531 brevets, 72 (soit 13,55\% seulement) sont déposés à plusieurs noms : une femme et un homme ou plusieurs femmes. Le dépôt de brevet est essentiellement une démarche individuelle.

Parmi ces inventrices, une large majorité de femmes mariées : 


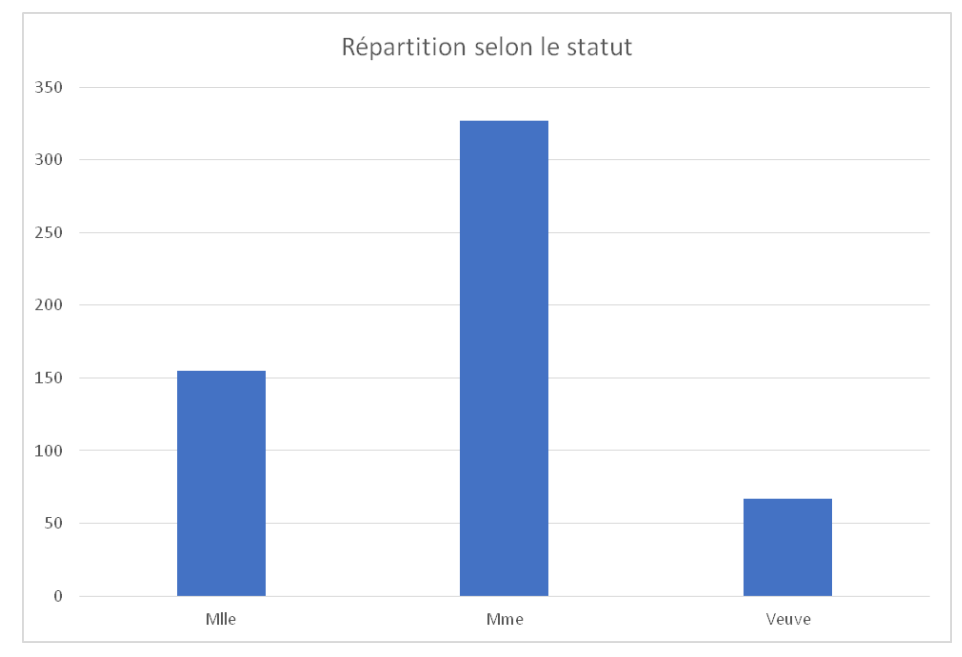

Il est étonnant de constater le nombre nettement inférieur de veuves et de célibataires, celles-ci ayant davantage de liberté juridique que les femmes mariées. Par ailleurs les veuves reprennent fréquemment l'entreprise du mari décédé, or seuls 6 statuts « société veuve» ont été relevés sur cette période.

Les domaines dans lesquels les femmes déposent des brevets correspondent à une classification de l'INPI qui répartit les inventions dans toutes les classes de l'activité industrielle :

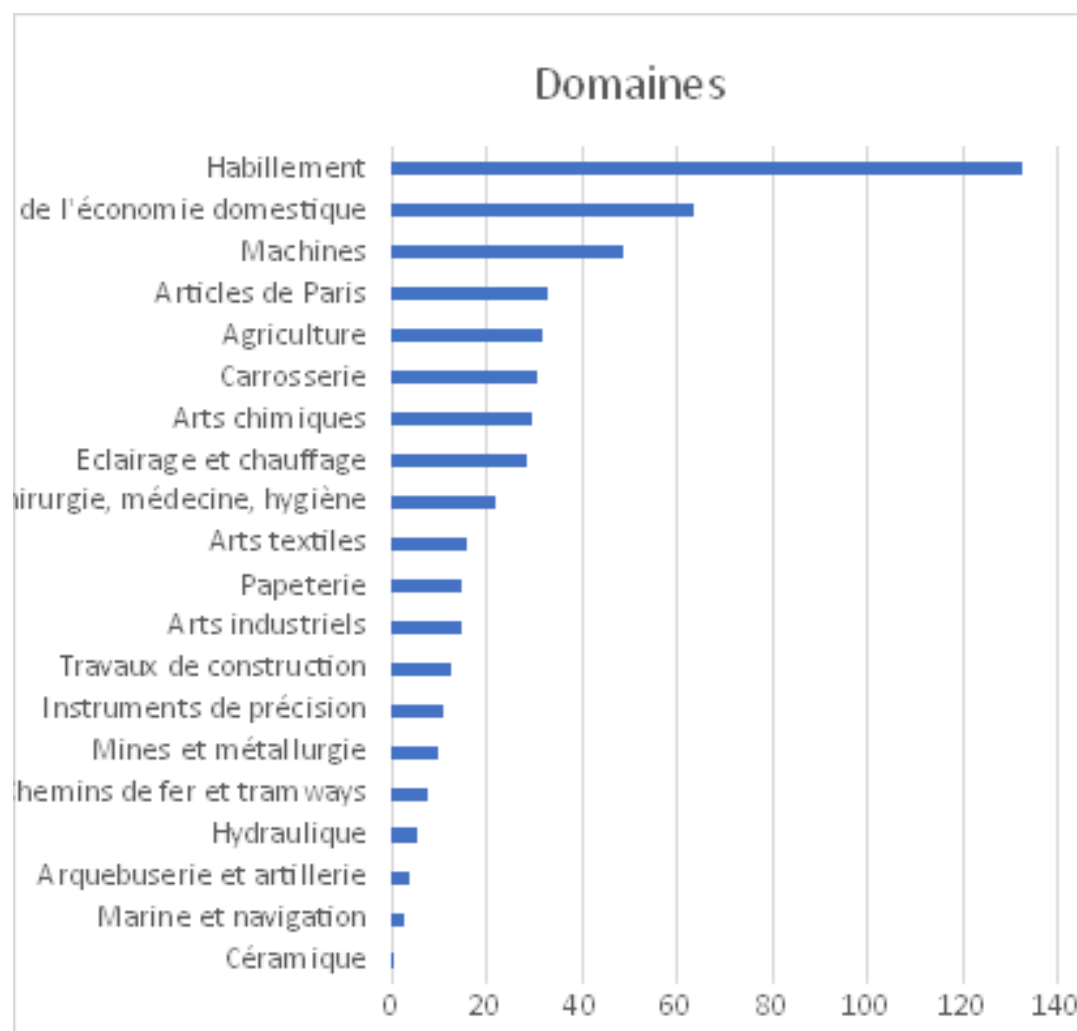

Le domaine habillement totalise à lui seul un quart des dépôts $(25,33 \%)$. Si on y ajoute les arts textiles, le pourcentage passe à $28,6 \%$ or, en 1900 , ces 2 domaines totalisent, tous brevets confondus, 7,53\% des brevets [GAL 04]. En poursuivant la comparaison grâce aux données de Gabriel Galvez-Behar, on constate ailleurs des similitudes: l'économie domestique et la consommation courante représente $22,07 \%$ de tous les brevets, $26,55 \%$ chez les femmes. Il n'est guère surprenant de les retrouver dans ce secteur : "L'économie domestique était devenue, au cours du XIXe siècle, une discipline autonome, pratiquée essentiellement par des femmes » [CAR 97]. En 
revanche le troisième domaine, en nombre de brevets déposés, peut paraître plus insolite : le domaine «machines » représente $9,33 \%$ des dépôts, $11,29 \%$ si on y ajoute les instruments de précisions pour comparer avec les données de Gabriel Galvez-Behar qui donne 19,69\% pour ces deux domaines ensemble en 1900. Le fait est que les femmes déposent des brevets dans toutes les classes de l'industrie et que les comparaisons de chiffres ne montrent pas de grandes distorsions hormis dans le secteur de l'habillement. Les femmes inscrivent leur activité inventive dans les secteurs en développement de la société.

L'obligation de mise en œuvre du brevet, d'exploitation de l'invention, est inscrite dans la loi. Les inventrices ont donc dû entrer dans un processus d'innovation : produire et commercialiser leur invention pour ne pas perdre le bénéfice du brevet. Cette partie fondamentale est en friche : l'INPI, consulté, ne possède pas d'informations sur le devenir des brevets. Deborah J. Warner remarque que « en 1900, 75\% des brevets accordés à des femmes durant les cinq années antérieures avaient la réputation de rapporter des retours profitables. Des inventions féminines présentées au Centennial, au moins 35\% étaient en réalité produites » [TRE 79]. De son côté Zorina Khan a étudié l'usage que des femmes ont fait de leurs brevets: elles vendent leurs droits, accordent des licences d'exploitation et développent des entreprises pour promouvoir leurs inventions. En ce qui concerne les femmes françaises déposant en France, elle a étudié le parcours de certaines d'entre elles entre 1791 et 1855 et conclu que ces femmes « introduisent des innovations, ont pris des risques, étaient actives dans la commercialisation et la concurrence, et la gestion des grandes entreprises » [TRE 79].

\section{Conclusion}

On ne peut guère tirer de conclusions du petit corpus qu'a permis d'obtenir le dépouillement de La Fronde mais l'étude produite à plus grande échelle (de 1791 à 1900) permettra sans doute d'éclairer plus largement et sûrement le rôle qu'ont tenu les femmes dans le monde de l'invention via les brevets. L'inventeur n'est pas un individu isolé, coupé d'une communauté de techniciens, de savants ainsi que de la connaissance de l'état des techniques et des sciences : il communique avec le milieu originel, culturel de son invention en tant qu'objet[30]. Il appartient à des cercles de connaissances, partage et se nourrit de savoirs. Une étude prosopographique permettrait de suivre l'origine, le parcours de certaines femmes, le devenir de leurs brevets. Ces femmes ont dû être actives tant dans leur milieu professionnel que dans les milieux juridiques : plusieurs ont déposé leurs brevets accompagnées par un mandataire, ingénieur civil, et certaines ont été confrontées à des procès en contrefaçon. Nous savons en outre qu'elles ont sollicité l'examen des académies, des sciences et de médecine, de la Société d'encouragement pour l'industrie nationale, afin de défendre leur invention, d'obtenir un encouragement et une validation.

Jusqu'à présent, les historiens, les sociologues, les féministes se sont interrogés sur les pratiques de la technique mais peu sur la connaissance des techniques par les femmes et leur implication dans le processus de l'invention. L'étude des brevets permet d'ouvrir un nouveau chapitre de l'histoire des femmes et de compléter celle des techniques.

\section{Bibliographie}

[ARM 93] Armengaud Eugène, Traité pratique des brevets d'invention, droits et obligations de l'inventeur et du breveté. Paris, France: Armengaud aîné, 1893.

[BEA 16] Beauvalet Scarlett, Berthiaud Emmanuelle, Le rose et le bleu: la fabrique du féminin et du masculin: cinq siècles d'histoire. Paris: Belin, 2016. 
[BEN 97] Bensaude-Vincent Bernadette, Rasmussen Anne, Éd., La science populaire dans la presse et l'édition: XIXe et XXe siècles. Paris, France: CNRS éd, 1997.

[CON 84] Constant Charles, Quelques Notes juridiques sur les brevets d'invention à l'usage des industriels, fabricants et commerçants. Paris, France: A. Chérié, 1884.

[CAR 97] Caron François, Les deux révolutions industrielles du XXe siècle. Paris, France: Albin Michel, 1997.

[DIZ 92] Dizier-Metz Annie, La Bibliothèque Marguerite Durand: histoire d'une femme, mémoire des femmes. Paris, France: Mairie de Paris, Direction des affaires culturelles: Bibliothèque Marguerite Durand, 1992.

[EMP 02] Emptoz Gérard, Marchal Valérie, Aux sources de la propriété industrielle guide des archives de l'INPI. Paris: INPI, 2002.

[FER 93] Ferry Jules, Discours et opinions de Jules Ferry. Tome premier, Le second Empire, la guerre et la Commune. Paris, France: A.Colin et cie, 1893.

[GAL 04] Galvez-Behar Gabriel, «"Pour la fortune et pour la gloire": inventeurs, propriété industrielle et organisation de l'invention en France, 1870-1922 », Thèse de doctorat, Université Charles de Gaulle, Lille ; 1971-2017, France, 2004.

[GAL 06] Galvez-Behar Gabriel, «Des médiateurs au sein du système d'innovation : les agents de brevets en France, 1870-1918 », in Archives de l'invention (les) : écrits, objets et images de l'activité inventive, des origines à nos jours, Toulouse, France: CNRS Université, 2006.

[GER 16] Gerhard Ute, « Droit civil et genre en Europe au XIXe siècle », Clio Femmes Genre Hist., n 43, p. 250-273, juin 2016.

[HIL 00] Hilaire-Pérez Liliane, Roche Daniel, L'invention technique au siècle des Lumières. Paris, France: Albin Michel, 2000.

[HIL 06] Hilaire-Pérez Liliane, «Invention technique et corpus de sources : identifier des pratiques, définir des contextes d'énonciation, analyser des représentations », in Archives de l'invention (les) : écrits, objets et images de l'activité inventive, des origines à nos jours, Toulouse, France: CNRS Université, 2006.

[JAC 15] Jacomy Bruno, Une histoire des techniques. Paris: Éd. Points, 2015.

[JAF 05] Jaffe Deborah, «Ingenius women », The CIPA journal (Chartered Institute of patent agents), $\mathrm{p}$. 285-290, mai-2005.

[KHA 05] Khan Zorina, The democratization of invention: patents and copyrights in American economic development, 1790-1920. Cambridge New York: Cambridge University Press, 2005.

[KHA] Khan Zorina, «Invisible women : entrepreneurship, innovation and family firms in France during early industrialization ». Working paper 20854, janv-2015.

[KLE 01] Kleinert Anne-Marie, Le "Journal des Dames et des Modes » ou la conquête de l'Europe féminine: 1797-1839. Stuttgart, Allemagne: J. Thorbecke, 2001.

[LAM 81] Lami Eugène-Oscar, Tharel Alfred, Dictionnaire encyclopédique et biographique de l'industrie et des arts industriels..., 8 vol. Paris, France: Lami, 1881.

[LEB 99] Lebeaume Joël, L'éducation technologique: histoires et méthodes. Paris, France, 1999.

[LEM 99] Leymarie Michel, La France contemporaine. De la Belle époque à la Grande Guerre: le triomphe de la République (1893-1914). Paris, France: Librairie générale française, 1999.

[PER 12] Perrot Michèle, Les femmes ou les silences de l'histoire. Paris: Flammarion, 2012.

[PIC 02] Picard Alfred, Exposition universelle Internationale de 1900 à Paris: rapport général administratif et technique. Tome premier. Paris, France: Imprimerie nationale, 1902.

[RAB 96] Rabaud Jean, Marguerite Durand (1864-1936) : "La Fronde» féministe ou "Le Temps » en jupons. Paris: L'Harmattan, 1996. 
[SCH 02] Schweitzer Sylvie, Les femmes ont toujours travaillé: une histoire de leurs métiers, XIXe et XXe siècle. Paris, France: Editions Odile Jacob, 2002.

[SIM 05] Simondon Gilbert, Chateau Jean-Yves, L'invention dans les techniques: cours et conférences. Paris: Seuil, 2005.

[SOH 96] Sohn Anne-Marie, Chrysalides: femmes dans la vie privée (XIXe-XXe siècles). Paris: Publications de la Sorbonne, 1996.

[THE 07] Thébaud Françoise, Corbin Alain, Écrire l'histoire des femmes et du genre. Lyon, France: ENS éditions, 2007.

[TRE 79] Trescott Martha Moore, Éd., Dynamos and virgins revised: women and technological change in history: an anthropology. Metuchen, NJ, Royaume-Uni de Grande-Bretagne et d'Irlande du Nord, 1979.

[VAN 99] VanœDulken Stephen, British patents of inventions 1617-1977:: a guide for researchers. London: British Library, 1999. 